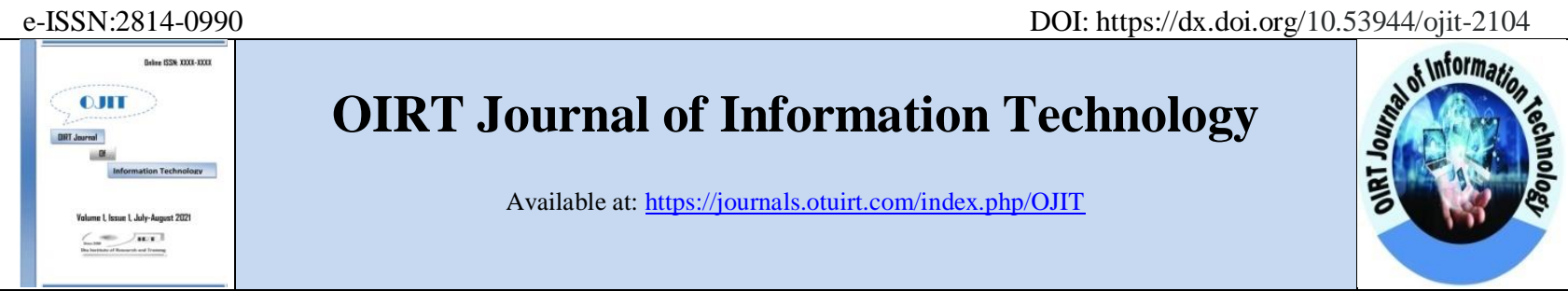

ORIGINAL RESEARCH ARTICLE

\title{
Efficient Machine Learning-Based Model for Cerebrovascular Disease Prediction with Nigerian Dataset
}

\author{
Lamido Yahaya $^{1 *}$, Gregory M. Wajiga ${ }^{2}$, Etemi J. Garba ${ }^{2}$ \\ ${ }^{1}$ Department of Computer of Science, Gombe State University, PMB 127, Gombe, Nigeria \\ ${ }^{2}$ Department of Computer Science, Modibbo Adama University, PMB 2076, Yola, Nigeria
}

Corresponding Author: Lamido Yahaya, Department of Computer of Science, Gombe State University, P.M.B. 127, Gombe, Nigeria. Email: yahayalmd@gsu.edu.ng

Copyright: (C) the author (s), publisher, and licensee OIRT. It is an open-access article distributed under the terms of the Creative Commons Attribution Non-Commercial License, which permits unrestricted non-commercial use, distribution, and reproduction in any medium, provided there is a proper citation for the original work.

\section{ABSTRACT}

Cerebrovascular accidents (CVA) or stroke has been a global phenomenon that causes disability and deaths of people worldwide, particularly in the middle- and low-income countries. It has been reported that more than 100,000 cases are recorded every year in Nigeria. Moreover, several deaths were reported globally by the World Health Organization (WHO). Diagnostic tools, preventive measures, and medical experts are insufficient and contribute to the escalation of the disease worldwide. Several predictive models have been proposed by scholars but have been inadequate due to variability in the risk factors, race, and geographical variations. This paper compared six machine learning-based models with three feature selection algorithms on a Nigerian dataset containing 103 instances with 22 features. We trained and evaluated the NB, SVM, LR, MLP, J-48, and RF with CBFS, CAE FS, and Relief FS algorithms. The results of our experiments showed that the J-48 model with the CBFS algorithm was computationally faster and achieved an excellent prediction accuracy of $100.00 \%$ in 0.00 seconds. The type of data used has a substantial impact on the performance of machine learning classifiers. Therefore, based on the experiments performed, J-48 with CBFS algorithm was proposed for deployment as the clinical decision support system that could assist medical professionals in predicting cerebrovascular diseases.

Keywords: Cerebrovascular Disease, Feature Selection, Machine Learning, Prediction, Predictive Models

\section{Article History}

Received: October 21, 2021

Revised: November 28, 2021

Accepted: December 01, 2021

\section{INTRODUCTION}

Cerebrovascular accidents (CVA) or stroke is one of the cardiovascular diseases (CVDs), which are among the leading causes of many deaths globally. It is a condition that affects the flow of blood and the blood vessels in the brain. It damages the brain due to blood supply interruption due to narrowing blood vessels or clot formation. The UI College of Medicine Report (2021) states that more than 100,000 cases are reported annually in Nigeria. It occurs either by a blocked artery (ischemic stroke) or blood vessel bursting 
(hemorrhagic stroke). An article by the Mayo Clinic Foundation stated that in transient ischemic attack (TIA), the brain's temporary disruption of blood flow occurs, which does not cause lasting symptoms (Mankad, 2021). According to the WHO (2011), in 2008, out of the 17.3 million CVD deaths, heart attacks were responsible for 7.3 million, and strokes were responsible for 6.2 million. The WHO reported that 17.9 million people die each year from CVDs. An estimated $31 \%$ of all deaths worldwide, $85 \%$, are due to heart attack and stroke (WHO, 2017).

However, a large portion of the disease occurs in low and middle-income countries where poorly structured healthcare systems. As a result, several cases and deaths occur in such countries without proper diagnoses and records. Therefore, accurate and early predictions are essential in preventing stroke from minimizing the number of cases and deaths worldwide. According to Hamman (2014), in all the various fields of prediction in CVAs, three significant variables are always important: age of the patient, severity, and subtype of the stroke. Though there are no clear boundaries between the three types of stroke (Adam et al., 2016), certain risk factors increase a person's chances of getting CVA. They include uncontrolled high blood pressure, diabetes or high blood sugar, heart diseases, smoking, family history, alcohol and drug misuse, poor diet, physical inactivity, obesity, high cholesterol, and age.

Nowadays, machine learning techniques are used to develop intelligent systems that efficiently support clinical decisions to predict diseases. The methods are used in a wide variety of applications, including healthcare. Clinical decision support systems are developed to predict conditions easily. A vast amount of data is generated by medical diagnoses every year. According to Wang \& Summers (2012), machine learning techniques play a vital role in radiological applications by automatically identifying complex patterns and helping radiologists make intelligent decisions. Despite the enormous development in the computer industry, the large amount of medical data has not been transformed into digital format in such sophisticated techniques for effective utilization. For instance, in Nigeria, the medical data is mainly kept manually in patients' folders, which requires extensive effort to extract, preprocess, and convert into helpful information for machine learning tasks. It's timeconsuming and tedious to transform the millions of manual data records available at the various medical institutions. That was the motivation behind conducting this research based on the CVA data from the Nigerian medical domain.

\section{RELATED WORKS}

This study presented some of the most recent machine learning-based research conducted for predicting cerebrovascular diseases.

Yu et al. (2020) proposed an AI-based system that detects stroke using real-time Electromyography (EMG) data. They employed the Random Forest algorithm in machine learning and Long Short-Term Memory (LSTM) to deeply understand the task. The EMG data were collected between 2015 and 2017 at the emergency medical center and the department of rehabilitation medicine at Chungnam National University Hospital, China. The data collected was for patients 65 age and above. The study obtained two hundred eighty-seven strokes and 271 normal individuals with 28 attributes. The predictive models were developed based on daily activities. Prediction accuracies of $90.38 \%$ and $98.958 \%$ were obtained for the RF and LSTM models, respectively.

Revant et al. (2020) performed a comparative study on Support Vector Machine (SVM), Decision Tree (DT), Random Forest (RF), and Multilayer Perceptron (MLP) in the prediction of CVA using a dataset that was not specified. Based on their experimental results, SVM achieved $98.99 \%$ accuracy in predicting the CVA or stroke disease.

Pathanjali \& Priya (2020) proposed a machine learning method, Support Vector Machine and Random Forest algorithms, to predict the risk after the first incidence of ischemic stroke. Models were validated using the 10 -fold cross-validation method. The compared algorithms achieved $98.45 \%$ and 98.44\% prediction accuracy for SVM and RF.

Zhu et al. (2021) conducted a study to predict CVA and understand its prevalence and influencing factors among residents in Qingyunpu District, Nanchang City, Jiangxi Province, China. A total of 2147 residents were considered a sample comprising 40 years and above during April and December 2018. They used Chi-square and Wilcoxon rank-sum tests for trend analysis and comparison of the risk level and employed Poisson regression for developing the predictive model. The model evaluation criteria used include specificity, sensitivity, receiver operating 
characteristic (ROC), and area under the curve (AUC). Based on their experimental results, the CVA prevalence was $4.5 \%$ among the participants, and the influencing factors include old age, hypertension, physical inactivity, smoking, diabetes, and family history of the disease.

Chun et al. (2021) compared Cox models, machine learning, and ensemble models in predicting cerebrovascular disease using 503842 adults in China between 2004 and 2008. First, they reached discrimination and calibration of Cox regression, support vector machine (SVM), logistic regression (LR), random forest (RF), multilayer perceptron (MLP), and gradient boosted tree (GBT) against the 2017 Framingham stroke risk score. Then, an ensemble model was developed to identify those at high risk of the disease. By applying the GBT or Cox model and based on individual-level characteristics, people at high risk of CVA, greater than $10 \%$, were predicted to have a 9 -year risk of the disease. For this risk (9-year), the ensemble approach gave higher accuracy of $76 \%$ for men and $80 \%$ for women, specificity of $76 \%$ for men and $81 \%$ for women.

Abedi et al. (2021) conducted a study to investigate whether machine learning algorithms could be trained to predict the recurrence of CVA and identify the key clinical features for the prediction and assess whether performance metrics could be optimized. The data of 2091 ischemic stroke patients with 53 attributes were obtained from the Geisinger E.H.R. system, Geisinger Quality database, and Social Security death database from September 2003 to May 2019. Six machine learning algorithms, including extreme gradient boosting, logistic regression, random forest, gradient boosting machine, support vector machine, and decision tree, where used with four feature selection techniques, five prediction windows, and two sampling techniques to develop 288 models for a 5year CVA recurrence prediction. Experimental results showed that from the 288 models, 21 models achieved an AUROC above 0.73. In comparison, 110 models achieved an AUROC greater than 0.7. Of the 53 features, age, high-density lipoprotein, body mass index, hemoglobin, and creatinine have the highest scores for the risk prediction.

Sailasya \& Kumari (2021) developed machine learning models based on Decision Trees, Logistic Regression, Random Forest, Naïve Bayes, Support Vector Machine, and K-Nearest Neighbor algorithms to predict CVA. It was a comparative study with the Kaggle dataset with 5110 instances and 12 features. From the results of their experiment, Naïve Bayes achieved $82 \%$ prediction accuracy.

Moreover, the reality in CVA prediction is that the risk factors vary, and geographical variations vary from one race to another. Therefore, predictive models developed mainly for one region may not suit another. That has created a gap in research for us to conduct a study based on a dataset obtained from Nigeria, where efficient diagnostic tools for cerebrovascular disease are insufficient. Nevertheless, this study helped us understand the high-risk factors of the disease in the sub-region and developed a predictive model accordingly.

\section{METHODOLOGY}

The architecture of the proposed CVA prediction model consists of data collection, data preprocessing, feature selection, cross-validation method, training of algorithms, CVA classification, and then model evaluation (Figure 1).

\section{The CVA Dataset Description:}

This dataset comprises 105 instances and 30 basic CVA diagnostic features with missing values. The data was collected with the Ethical Clearance granted on May 19, 2021, by the Research and Ethics Committee of the Federal Teaching Hospital, Gombe, Nigeria. The data was extracted from patients' folders at the Health Records unit of the medical institution. Of the 105 instances, 49 were males, and 56 were females. The breakdown of male-female cases was illustrated in Figure 2, and the detailed descriptions of the various features of the dataset were presented in Table 1. 


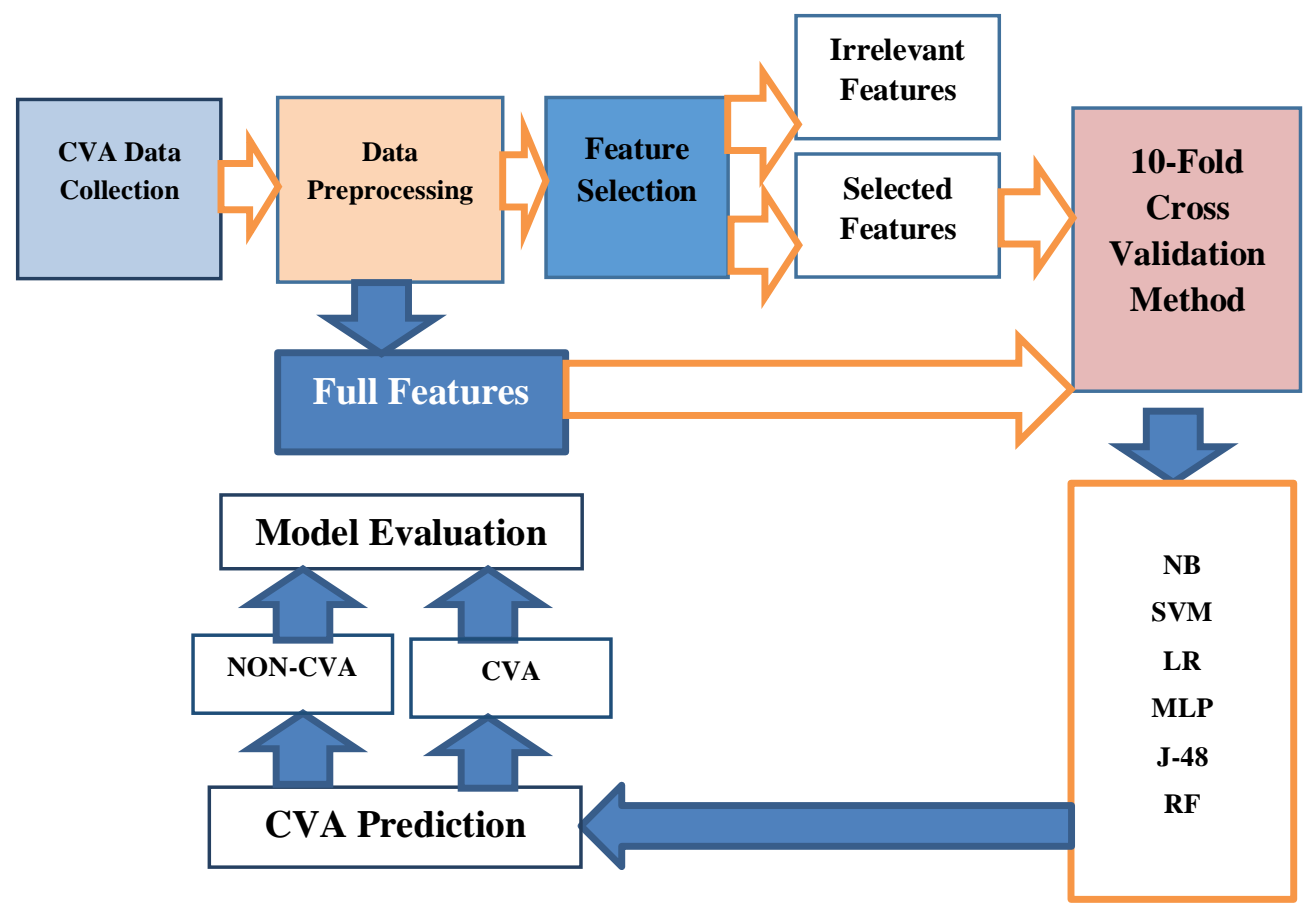

Figure 1. Intelligent Framework for CVA Prediction

Based on the description of the CVA dataset in Table 1 , the class attribute indicates that from the total of 105 patients (both males and females), 77 patients were diagnosed as CVA positive. In contrast, 28 were diagnosed or classified as CVA negative (Figure 3).

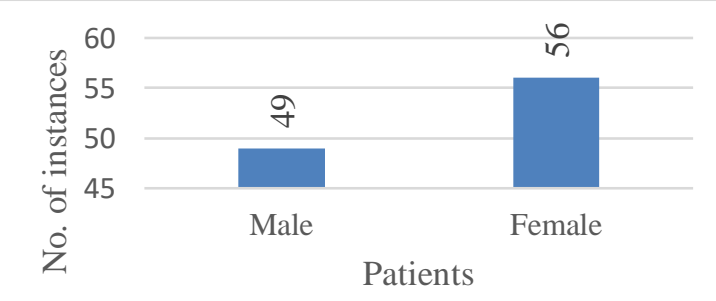

Figure 2. Male-Female C.V.A. Cases at FTH Gombe from 2010 to 2020

\section{Dataset Preprocessing:}

The dataset collected contains inconsistencies such as missing values, irrelevant features, and noise. Therefore, necessary steps for data cleaning, such as preprocessing, were applied to the sample to ensure the cleanness of the sample collected. Therefore, classification accuracy is highly dependent upon the type of datasets used.
The original CVA dataset contains 105 instances with 30 raw attributes or features. Unfortunately, the sample has some missing values and irrelevant or less relevant features. Out of the 30 diagnostic features of the sample, 'ID,' 'AGE,' and 'SEX' is related to patients' personal information, while 'NUM' is the target or class attribute. The remaining 26 features are patients' clinical information recorded during the medical examination. However, two instances and three points (the columns for 'WEIG.' 'FH,' and 'RBS') were removed due to the high frequency of missing values, while 'ID,' 'TMP.,' 'TGL,' 'ESR,' and 'WBC' were removed due to less relevancy. Therefore, two instances and eight features were removed in all. The 'WEIG' and 'FH' attributes strongly connect with CVA but were removed due to many missing values. For example, most patients could not stand for possible weight measurement during medical examinations. So, in most cases, we didn't record the attribute. The final preprocessed sample contains 103 instances with 21 CVA diagnostic features and 1 class attribute.

\section{Building the Models:}

The popular java-based machine learning tool, Waikato Environment for Knowledge Analysis 
(WEKA), was used for the experimental setup. The models were built based on some popular algorithms, which include Naïve Bayes (NB), Support Vector Machine (SVM), Logistic Regression (LR), Multilayer Perceptron (MLP), Decision Tree (J48DT), and Random Forest (RF). The objective was to determine which classifiers could better predict the CVA disease based on the obtained dataset.

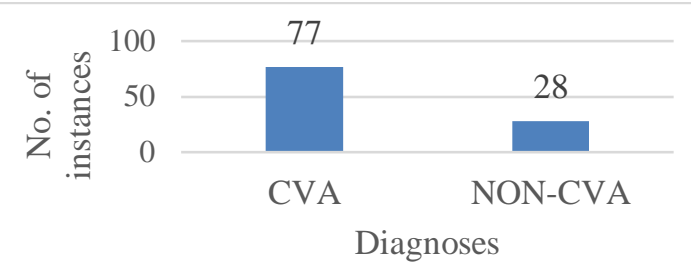

Figure 3. The CVA Positive and Negative Cases

Table 1: Descriptions of the Dataset

\begin{tabular}{|c|c|c|}
\hline Feature & Description & Type \\
\hline ID & A six-digit patients' unique hospital number & Numeric \\
\hline AGE & Age of diagnosed patients (in years) & Numeric \\
\hline SEX & $\begin{array}{l}\text { Gender of diagnosed patients with two values: } \\
\text { Value 1: Male } \\
\text { Value 0: Female }\end{array}$ & Nominal \\
\hline $\mathrm{BP}$ & Resting Blood Pressure of patients (in $\mathrm{mmHg}$ on admission to the hospital measured) & Numeric \\
\hline $\mathrm{FH}$ & $\begin{array}{l}\text { Patient's Family History of CVA with two values: } \\
\text { Value 0: indicates no history of CVA in the patient's family } \\
\text { Value 1: shows the history of CVA in the patient's family }\end{array}$ & Nominal \\
\hline PR & Pulse Rate or heartbeats of patients (in bpm on admission to the hospital) & Numeric \\
\hline CS & $\begin{array}{l}\text { Patients' chest status upon examination with two values: } \\
\text { Value 1: indicates the presence of a chest problem } \\
\text { Value } 0 \text { : shows the absence of chest problem }\end{array}$ & Nominal \\
\hline BWK & $\begin{array}{l}\text { Body weakness, either left or right of a patient on admission to the hospital, with two } \\
\text { values: } \\
\text { Value 1: indicates the presence of left/right weakness } \\
\text { Value 0: shows the absence of the weakness }\end{array}$ & Nominal \\
\hline TMP & Patient's body temperature on admission to the hospital (in degrees Celsius) & Numeric \\
\hline RS & Patient's respiratory counts (in rpm on admission to the hospital) & Numeric \\
\hline WBC & Patient's white blood cell counts after laboratory test & Numeric \\
\hline ESR & Erythrocyte sedimentation rate test result (in $\mathrm{mm} / \mathrm{hr}$.) & Numeric \\
\hline TCL & Patient's total cholesterol (in $\mathrm{mmol} / \mathrm{L}$ ) & Numeric \\
\hline HDL & High-density lipoprotein or good cholesterol (in $\mathrm{mmol} / \mathrm{L}$ ) & Numeric \\
\hline LDL & Low-density lipoprotein or bad cholesterol (in $\mathrm{mmol} / \mathrm{L}$ ) & Numeric \\
\hline TGL & Triglycerides (in mmol/L) & Numeric \\
\hline FBS & Fasting blood sugar of patients (in $\mathrm{mmol} / \mathrm{L}$ ) & Numeric \\
\hline RBS & Random blood sugar (in mmol/L) & Numeric \\
\hline SOD & Total sodium ions present in the patient's plasma (in $\mathrm{mmol} / \mathrm{L}$ ) & Numeric \\
\hline POT & Total potassium ions present in the patient's plasma (in $\mathrm{mmol} / \mathrm{L}$ ) & Numeric \\
\hline CHL & Total chloride ions present in the patient's plasma (in $\mathrm{mmol} / \mathrm{L}$ ) & Numeric \\
\hline $\mathrm{BCB}$ & Total hydrogen bicarbonate ions present in the patient's plasma (in $\mathrm{mmol} / \mathrm{L}$ ) & Numeric \\
\hline U.R. & Urea level in patient's plasma (measured in $\mathrm{mmol} / \mathrm{L}$ ) & Numeric \\
\hline CR. & Creatinine level in patient's plasma (measured in $\mathrm{mmol} / \mathrm{L}$ ) & Numeric \\
\hline UA. & The uric acid level in the patient's plasma (measured in $\mathrm{mmol} / \mathrm{L}$ ) & Numeric \\
\hline NTP & Neutrophils count (measured in $\mathrm{mm}^{3}$ ) & Numeric \\
\hline LYM & Lymphocytes count (measured in $\mathrm{mm}^{3}$ ) & Numeric \\
\hline MON & Monocytes count (measured in $\mathrm{mm}^{3}$ ) & Numeric \\
\hline WEIG & Patient's weight (measured in $\mathrm{kg}$ ) on admission to the hospital & Numeric \\
\hline NUM & $\begin{array}{l}\text { Diagnosis of CVA contains two values: } \\
\text { Value } 1 \text { (CVA): the presence of CVA disease } \\
\text { Value } 0 \text { (NON-CVA): absence of CVA disease }\end{array}$ & Nominal \\
\hline
\end{tabular}




\section{Feature Selection:}

In their work, Kiruthikaa et al. (2018) mentioned that the "feature selection algorithm has the highest impact on determining the prediction accuracy rates besides the selection of learning techniques alone." The same technique's prediction accuracy varies according to feature sets and several patient instances (Haq et al., 2018). Following feature selection techniques were used in the experiment.

\section{i. Correlation Based Feature Selection (CBFS) Algorithm}

The CBFS algorithm is a popular technique for selecting the most relevant attributes in a dataset. First, it calculates the correlation between each point and the output variable. Then, it sets only those attributes with a moderate to a high positive or negative correlation close to -1 or 1 . It will then drop those attributes with low correlation values (i.e., values close to zero). So this technique is supported by WEKA, and it requires the use of the Ranker search method.

\section{ii. Classifier Attribute Evaluator Feature Selection (CAE FS) Algorithm}

The CAE algorithm evaluates the worth of an attribute by measuring the impact of leaving it out from the complete set instead of considering its price in isolation. This technique is also supported by WEKA and requires the Ranker search method.

\section{iii. Relief Attribute Evaluator Feature Selection (RAE FS) Algorithm}

This technique evaluates the worth of an attribute by repeatedly sampling an instance and considering the value of the given point

Moreover, from the confusion matrix (Table 2), the following metrics were computed, and our models were evaluated based on accuracy, sensitivity, precision, MCC, ROC, and processing time. They are as follows:

$\mathbf{T P}=$ True Positive, the condition where the number of instances classified as accurate was actual. The model organized the case where a person has a CVA. for the nearest example of the same and different class. RAE can operate on both discrete and continuous class data and also requires the use Ranker search method in WEKA.

\section{Cross-Validation:}

A stratified cross-validation approach was applied to validate the classifiers at this stage. Specifically, a kfold cross-validation method was employed. In this research, ten was selected as the value of $k$, that is, $k=$ 10. "This is called 10-fold cross-validation" (Smola \& Vishwanathan, 2010). The value ten was chosen for better performance. $90 \%$ of the data was used to train the algorithms in this process, while $10 \%$ was used for testing purposes. The process was repeated ten times for each fold to ensure that all instances participated in the training and testing phases. All training and testing set instances were randomly divided over the whole dataset. At the end of the 10-fold process, averages of all performance metrics were computed.

\section{CVA Model Performance Evaluation Method:}

Each of the models was evaluated based on various evaluation metrics. A confusion matrix was used, where every observation in the testing set is predicted in exactly one box. Since there are two classes in the datasets, the matrix used was $2 \times 2$ matrices. Therefore, it gives each classifier two correct and two incorrect predictions (Table 2).

Table 2: Confusion Matrix for CVA Prediction

\begin{tabular}{|l|c|c|c|} 
& & $\begin{array}{c}\text { Predicted } \\
\text { CVA patient } \\
(1)\end{array}$ & $\begin{array}{c}\text { A predicted } \\
\text { average } \\
\text { person (0) }\end{array}$ \\
\hline $\begin{array}{l}\text { Actual } \\
\text { patient (1) }\end{array}$ & C.V.A. & TP & FN \\
\hline $\begin{array}{l}\text { An actual average } \\
\text { person (0) }\end{array}$ & FP & TN \\
\hline
\end{tabular}

$\mathbf{T N}=$ True Negative, the condition where the number of instances classified as false while they were false. It is the case where a person does not have a CVA, and the model classified him as not having CVA. FP $=$ False Positive, the condition where the number of instances is classified as true while false. The model organized the case where a person does not have a CVA. 
FN = False Negative, the number of instances classified as false while true. It is the case where a person has a CVA but was organized by the model as not having a CVA.

Accuracy: Classification accuracy is a metric that shows the overall performance. It can be computed as follows:

$$
\text { Accuracy }=\frac{T P+T N}{T P+T N+F P+F N} \times 100 \%
$$

Sensitivity: Sensitivity, also called Recall, is a metric that shows what a model predicted proportion of patients with CVA as having CVA. The sensitivity of a model can be computed as follows:

$$
\text { Sensitivity }=\frac{T P}{T P+F N} \times 100 \%
$$

Precision: Precision is a metric that tells what proportion of patients that the model predicted as having a CVA had the CVA. The precision can be calculated as:

$$
\text { Precision }=\frac{T P}{T P+F P} \times 100 \%
$$

Mathew Correlation Coefficient (MCC): The MCC of a classifier represents its prediction ability values that, i.e., are within the range of -1 to +1 . The MCC of a classifier with the value of +1 means its predictions are ideal. Conversely, an MCC value of -1 indicates the wrong predictions of the available classifier, while deals close to 0 suggest that the available classifier generates random predictions.

$$
\text { MCC }=\times 100 \%
$$

Receiver Operating Characteristic (ROC.) Area: It is a graph that compares the true positive rate and falsepositive rate in the classification results of a model. It analyses the prediction capability of a model.

\section{RESULTS AND DISCUSSIONS}

This section conducted experiments by developing models using our dataset with the selected algorithms, including NB, SVM, LR, MLP, J48, and RF. In the first instance, the models were evaluated on the full features of the data. Secondly, three feature selection algorithms, CBFS, CAE FS, and RAE FS, were used to select more relevant predictors from the dataset. Then, the performances of the models were evaluated on the selected features. Finally, the performance evaluation metrics were used to check the performance of each classifier based on a 10-fold stratified crossvalidation method. All computations were performed automatically in the WEKA tool on an Intel(R) Pentium(R) CPU 2020M @ 2.40GHz PC.

Table 3: Classifiers Performances on full features

\begin{tabular}{|c|c|c|c|c|c|c|}
\hline Classifier & Accuracy (\%) & $\begin{array}{c}\text { Sensitivity } \\
(\%)\end{array}$ & $\begin{array}{c}\text { Precision } \\
(\%)\end{array}$ & MCC (\%) & ROC (\%) & $\begin{array}{c}\text { Execution } \\
\text { Time (s) }\end{array}$ \\
\hline NB & 96 & 96 & 97 & 90 & 100 & 0.14 \\
\hline SVM & 100 & 100 & 100 & 100 & 100 & 0.13 \\
\hline LR & 99 & 99 & 99 & 97 & 100 & 0.20 \\
\hline MLP & 99 & 99 & 99 & 97 & 95 & 0.41 \\
\hline J-48 & 100 & 100 & 100 & 100 & 100 & 0.02 \\
\hline RF & 100 & 100 & 100 & 100 & 100 & 0.03 \\
\hline
\end{tabular}

Training and Evaluation of Classifiers on Full Features:

In this experiment, the algorithms were trained and evaluated on the full features of the dataset. In the CVA dataset, $n=21$, where $n$ is the number of features. However, SVM, J-48, and RF models showed an excellent performance with 100\% classification accuracy, sensitivity, precision, MCC, and ROC. Meanwhile, the J-48 model has the shortest processing time of 0.02 seconds. In Table 3, the performances of each classifier and the results obtained were very good for almost all the selected classifiers were shown.

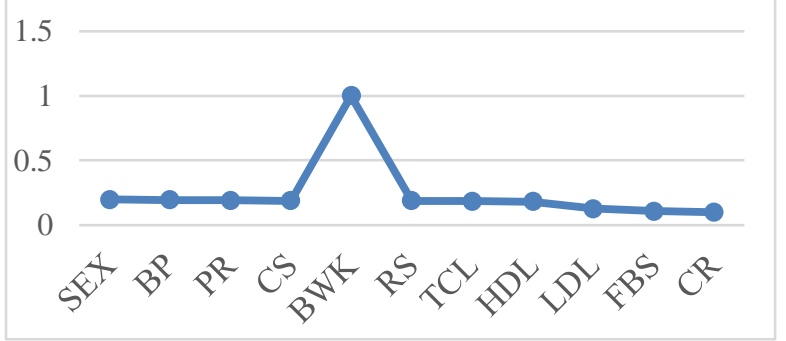

Figure 4. Feature Ranking by CBFS Algorithm 
Training and Evaluation of Classifiers on Selected Features:

Three different feature selection algorithms were employed to examine which feature selection technique would better select the most relevant predictors of cerebrovascular diseases. The algorithms used were Correlation Based Feature Selection (CBFS), Classifier Attribute Evaluator Feature Selection (CAE FS), and Relief Attribute Evaluator Feature Selection (RAE FS). The selected features in the dataset: $n=11$, where $n$ is the number of selected features. Each feature selection algorithm was used with the six selected classification algorithms.

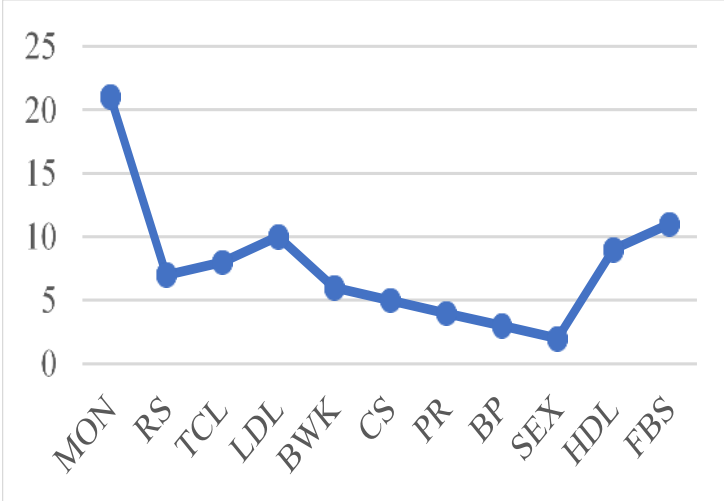

Figure 5: Feature Ranking by CAE algorithm

Table 4: Classifiers Performances on selected features by CBFS Algorithm

\begin{tabular}{|c|c|c|c|c|c|c|}
\hline Classifier & Accuracy (\%) & $\begin{array}{c}\text { Sensitivity } \\
(\%)\end{array}$ & Precision (\%) & MCC (\%) & ROC (\%) & $\begin{array}{c}\text { Execution } \\
\text { Time (s) }\end{array}$ \\
\hline NB & 97.09 & 97.10 & 97.10 & 92.40 & 98.70 & 0.00 \\
\hline SVM & 100.00 & 100.00 & 100.00 & 100.00 & 100.00 & 0.00 \\
\hline LR & 100.00 & 100.00 & 100.00 & 100.00 & 100.00 & 0.12 \\
\hline MLP & 100.00 & 100.00 & 100.00 & 100.00 & 100.00 & 0.16 \\
\hline J-48 & 100.00 & 100.00 & 100.00 & 100.00 & 100.00 & 0.00 \\
\hline RF & 100.00 & 100.00 & 100.00 & 100.00 & 100.00 & 0.03 \\
\hline
\end{tabular}

\section{Classifiers' Performances on Selected Features by} CBFS Algorithm:

The algorithm selected 11 features based on ranking. They include SEX, BP, PR, CS, BWK, RS, TCL, HDL, LDL, FBS, and CR. In the experiment, SVM, LR, MLP, J-48, and RF achieved $100 \%$ in all performance metrics, with varying computation times. SVM, J-48, and NB recorded 0.00 seconds to complete their tasks in terms of execution time. Therefore, there is an improvement in the performance of the CBFS algorithm. The feature ranking and classifier versions are shown in Figure 4 and Table 4, respectively.

\section{Classifiers' Performances on Selected Features by CAE FS Algorithm:}

The CAE feature selection algorithm also works with the Ranker search technique, where the most important features are given the highest priority. The algorithm does not assign any score to an attribute but ranks them in importance for the prediction. From the 21 diagnostic features of the dataset, the first 11 were selected. All the six classifiers except NB achieved $100 \%$ in all performance evaluation measures with varying computation times in the experiment. The ranking of features and classifier performances are shown in Figure 5 and Table 5, respectively.

Table 5: Classifiers Performances on selected features by CAE FS

\begin{tabular}{|c|c|c|c|c|c|c|}
\hline Classifier & $\begin{array}{c}\text { Accuracy } \\
(\%)\end{array}$ & $\begin{array}{c}\text { Sensitivity } \\
(\%)\end{array}$ & Precision (\%) & $\operatorname{MCC}(\%)$ & ROC (\%) & $\begin{array}{c}\text { Execution } \\
\text { Time (s) }\end{array}$ \\
\hline NB & 99.03 & 99.00 & 99.00 & 97.50 & 100.00 & 0.00 \\
\hline SVM & 100.00 & 100.00 & 100.00 & 100.00 & 100.00 & 0.00 \\
\hline LR & 100.00 & 100.00 & 100.00 & 100.00 & 100.00 & 0.00 \\
\hline MLP & 100.00 & 100.00 & 100.00 & 100.00 & 100.00 & 0.14 \\
\hline $\mathrm{J}-48$ & 100.00 & 100.00 & 100.00 & 100.00 & 100.00 & 0.00 \\
\hline $\mathrm{RF}$ & 100.00 & 100.00 & 100.00 & 100.00 & 100.00 & 0.03 \\
\hline
\end{tabular}


Classifiers Performances on Selected Features by Relief FS Algorithm:

The Relief FS algorithm works the same way as the CBFS algorithm by assigning scores to features based on priority. However, one of its drawbacks is that Relief does not work with datasets with many features like CVA, which has 21 parts. Therefore, the algorithm was subjected to 11 elements already reduced by other algorithms. Then, six were ranked the most important for predicting CVA. All classifiers except NB recorded $100.00 \%$ in all performance evaluation metrics with varying computation times in the experiment. NB, LR, and J-48 were computationally the fastest, with 0.00 seconds in execution. The feature ranking and classifier performances were presented in Figure 6 and Table 6 , respectively.

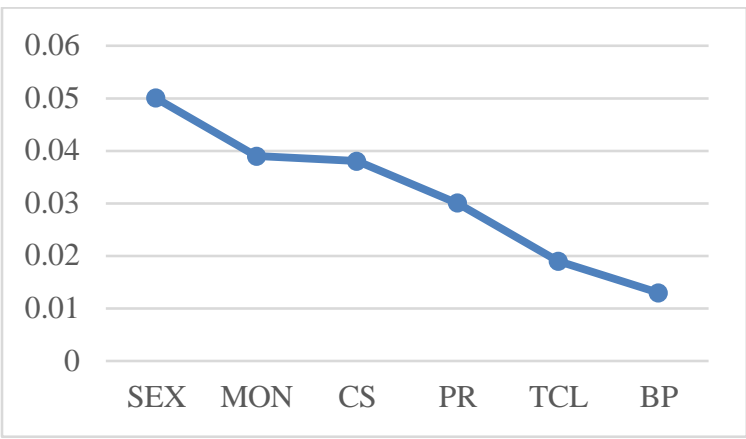

Figure 6. Feature Ranking by Relief FS algorithm

Table 6: Classifiers Performances on selected features by Relief

\begin{tabular}{|c|c|c|c|c|c|c|}
\hline \multirow{2}{*}{ Classifier } & Accuracy (\%) & $\begin{array}{c}\text { Sensitivity } \\
(\%)\end{array}$ & Precision (\%) & MCC (\%) & ROC (\%) & $\begin{array}{c}\text { Execution } \\
\text { Time (s) }\end{array}$ \\
\hline NB & 99.03 & 99.00 & 99.00 & 97.50 & 100.00 & 0.00 \\
\hline SVM & 100.00 & 100.00 & 100.00 & 100.00 & 100.00 & 0.02 \\
\hline LR & 100.00 & 100.00 & 100.00 & 100.00 & 100.00 & 0.00 \\
\hline MLP & 100.00 & 100.00 & 100.00 & 100.00 & 100.00 & 0.09 \\
\hline J-48 & 100.00 & 100.00 & 100.00 & 100.00 & 100.00 & 0.00 \\
\hline RF & 100.00 & 100.00 & 100.00 & 100.00 & 100.00 & 0.03 \\
\hline
\end{tabular}

\section{Comparing Classification Accuracies on Full and} Selected Features:

The SVM, J-48, and RF maintained $100.00 \%$ accuracy, while LR and MLP improved from $99.00 \%$ to $100.00 \%$, showing effectiveness with all the feature selection techniques. NB model improved from full features with CBFS and CAE FS but maintained the same performance with Relief FS. In terms of execution times, J-48 appeared more efficient with 0.00 seconds with all feature selections, and it was more efficient in predicting CVA diseases. All these and various computation times are shown in Figures 7 and 8 , respectively.



Figure 7. Comparing Accuracies of Classifiers on Full and Selected Feature

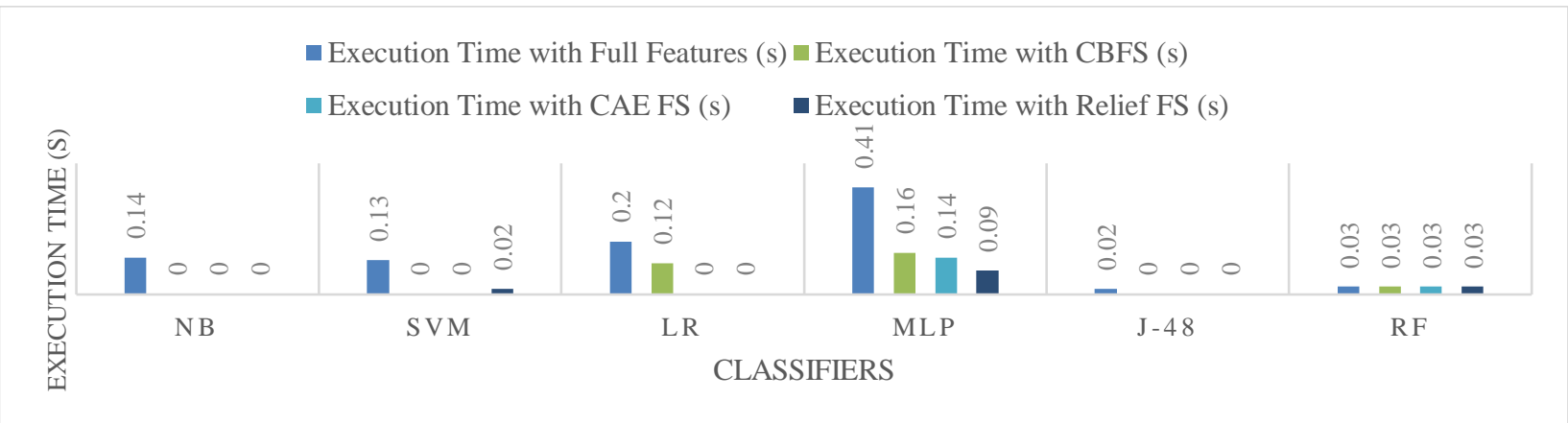

Figure 8. Execution Times of Classifiers with Full and Selected Features 


\section{CONCLUSION}

This paper conducted a comparative approach on six machine learning models with three feature selection techniques using Nigerian data to determine the most efficient classifier for predicting the CVA disease. The type of data used has a substantial impact on the performance of the classifiers. Therefore, based on the experiments performed, J-48 with CBFS algorithm performed excellently in predicting the disease and was proposed for implementation and deployment as the clinical decision support system that could assist medical professionals in predicting cerebrovascular diseases.

\section{Acknowledgments}

The authors wish to acknowledge and thank the management of the Federal Teaching Hospital (FTH.) Gombe for granting permission to facilitate the data collection at the medical institution.

Ethical Approval: Ethical Approval was granted by the Research \& Ethics Committee of the FTH Gombe on May 19, 2021

\section{Funding: None}

\section{Conflict of Interest: None declared}

\section{REFERENCES}

Abedi, V., Avula, V., Chaudhary, D., Shahjouei, S., Khan, A., Griessenauer, C. J., . . . ... (2021). Prediction of LongTerm Stroke Recurrence Using Machine Learning Models. Journal of Clinical Medicine. doi:10.3390/jcm10061286

Adam, S. Y., Yousif, A., \& Bashir, M. B. (2016). Classification of Ischemic Stroke using Machine Learning algorithms. International Journal of Computer Applications, 149(10), 26-31.

Chun, M., Clarke, R., Cairns, B. J., Clifton, D., Bennett, D., Chen, Y., ...... (2021). Stroke Risk Prediction using Machine Learning: a prospective cohort study of 0.5 million
Chinese adults. Journal of the American Medical Informatics Association, 28(8), 1719-1727. doi:10.1093/Jamia/ocab068

Hamman, G. (2014, October). Prediction in cerebrovascular diseases. Nervenarzt, German. doi:10.1007/s00115-0144063-1

Haq, A. U., Li, J.-P., Memon, M. H., Nazir, S., \& Sun, R. (2018). A hybrid intelligent system framework for predicting heart disease using machine learning algorithms. Hindawi Mobile Information System. doi:10.1155/2018/3860146

Kiruthikaa, K. V., Franklin, V. J., \& Yuvaraj, S. (2018). Analysis of prediction accuracy of heart diseases using supervised machine learning techniques for developing clinical decision support systems. International Journal of Recent Technology and Engineering, 7(4S), 433-437.

Mankad, R. (2021, February 27). Stroke. Retrieved from Mayo Clinic Foundation for Medical Education and Research: https://www.mayoclinic.org

Pathanjali, C., \& Priya, T. (2020). Machine Learning for Predicting Ischemic Stroke. International Journal Of Engineering Research and Technology, 9(5), 1334-1337.

Revant, S., Sanjay, S., Sanjay, N., \& Vijayaganath, V. (2020). Stroke Prediction using Machine Learning Algorithms. International Journal of Disaster Recovery and Business Continuity, 11(1), 3081-3086.

Sailasya, G., \& Kumari, G. L. (2021). Analyzing the Performance of Stroke Prediction Using ML Classification Algorithms. International Journal of Advanced Computer Science and Applications, 12(6), 539-545.

Smola, A., \& Vishwanathan, S. V. (2010). Introduction to machine learning. New York: Cambridge University Press.

UI College of Medicine Report. (2021). Angina: Symptoms and Treatments. Ibadan: University Press.

Wang, S., \& Summers, R. M. (2012). Machine learning and radiology. Medical Image Analysis, 16(5), 933-951.

WHO. (2011). Global Atlas on cardiovascular disease prevention and control. Geneva: WHO Library Cataloguing.

WHO. (2017). Global action plan for the prevention and control of noncommunicable diseases. Geneva: WHO Library Cataloguing.

Yu, J., Park, S., Kwon, H., Ho, C. M., \& Pyo, C. (2020). AI-Based Stroke Disease Prediction System Using Real-Time Electromyography Signals. Applied Sciences. doi:10.3390/app10196791

Zhu, Q., Luo, D., Zhou, X., Cai, X., Li, Q., Lu, Y., \& Chen, J. (2021). A Model for Risk Prediction of Cerebrovascular Disease Prevalence--Based on Community Residents Aged 40 and Above in a City in China. International Journal of Environmental Research and Public Health. doi:10.3390/ijerph18126584. 